\title{
Nano-To-Micro Self-Assembly Using Shear Flow Devices
}

\author{
${ }^{l}$ Chi-Yuan Shih, ${ }^{\text {ISiyang Zheng, }}{ }^{\text {E Ellis Meng, }}{ }^{\text {IY } Y \text {-Chong Tai, }}{ }^{2}$ Yi Liu and ${ }^{2} J$. Fraser Stoddart \\ ${ }^{1}$ Caltech Micromachining Laboratory \\ Electrical Engineering Department MC136-93, California Institute of Technology \\ Pasadena CA91125 USA \\ Telephone: (626)395-2227 Fax(626)584-9104 \\ E-mail:cyshih@caltech.edu \\ ${ }^{2}$ University of California Los Angeles, Department of Chemistry and Biochemistry
}

\begin{abstract}
This work aims at developing a new technique to precisely assemble nano-materials into micro- or even meso-scale devices. For example, our long-term goal is to use massively architected motor-molecules [1] to build muscle-like actuators in which these molecules work in parallel to output large forces. As an important first step, we report here the successful development of a much improved shear-flowenhanced self-assembly method over the baseline spontaneous assembly method in test tubes [2]. More specifically, we have engineered special thiolated model molecules (bisdisulfide $/ \mathrm{C}_{28} \mathrm{H}_{34} \mathrm{O}_{4} \mathrm{~S}_{4}$ ) and demonstrated the nano-to-micro self-assembly through flow interface using thiol-gold bonding chemistry. Our method has produced gold/molecule aggregates as big as $50 \mu \mathrm{m}$ that are completely made of $30 \mathrm{~nm}$ gold nanoparticles and $3 \mathrm{~nm}$ model molecules
\end{abstract}

\section{INTRODUCTION}

Precision molecular assembly is the cornerstone of nanotechnology. It follows that the major technical challenge is to assemble arrays of motor molecules repeatedly and coherently from the nano up to micron scale. As a first step, we will research a new technology to manufacture micronscale, muscle-like actuators from assemblies of nanoscale linear-motor molecules and gold nanoparticles. We propose to use nano/microfluidic technology to achieve this goal. Integrated microfluidic systems will be developed to manipulate strategically thiolated motor-molecules and gold nanoparticles. Our goal is to not only demonstrate the feasibility of fabricating biomimetic muscle fibers based upon engineered linear motor molecules, but to do so using scalable technology.

Fig. 1 shows the basic idea of our shear-flow assembly. The interface of two shear flows is where gold nanoparticles meet with the thiolated molecules, herein the aggregation happens. The important advantages of this approach are twofold. The first is to limit the assembly only at flow interface for controlled assembly. The second is the unsaturated growth of

aggregate because shear flows continue to supply fresh nanomaterials to the interface, leading to large aggregates.

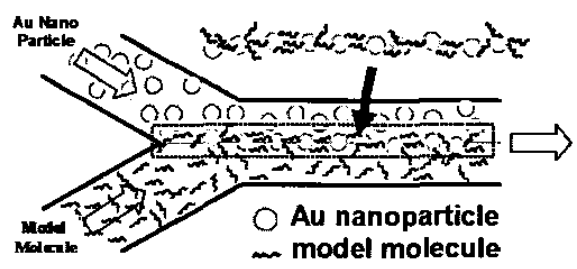

Figure 1: "Shear Flow Assembly" working principle.

\section{FABRICATION}

To achieve molecular assembly in microfluidic devices, we have designed and fabricated two types of devices as described in the following. The first type of devices is to make PDMS (Sylgard 184) molded channels bound to glass substrate as shown in Figure 2(a). Microchannels of $10 \mu \mathrm{m}$ deep are molded onto PDMS surface. The PDMS pieces are then bound to glass slides by overnight baking at $80^{\circ} \mathrm{C}$. A total of six designs with different number of input channels, channel widths and shear flow configurations are made to study their effects on assembly efficiency.

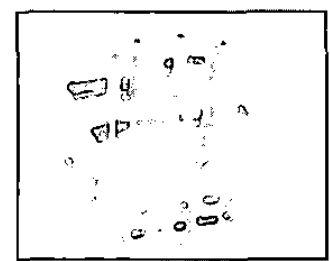

Figure 2: Devices fabricated. glass/silicon device.

Due to the fact that the proposed "muscle" molecules dissolve much better in organic solvents which in general are not compatible with PDMS, we design and fabricate a glass/silicon device as shown in Figure 2(b). Fluidic channels were formed on silicon wafer with DRIE. Anodic bonding is used to bond silicon chip to glass chip. This device is capable 
of handling any organic solvent including dichlomethane (DCM) and acetone that are not suitable for PDMS material.

\section{DEVICE AND REACTION SYSTEM CALIBRATION}

\section{Shear flow device calibration}

Once devices are made, syringe pumps are used for liquid supply with a wide range of flow rate controllability. As shown in Figure 3, the shear-flow width is defined to be the width of the central flow after the multiple inlet junctions (in the case of a three-input channel design). The central flow width calibration is done using fluorescence dye under a fluorescence microscope. Our results show that central flow width as small as $1 \mu \mathrm{m}$ or smaller can be achieved, which is perfect for muscle fiber synthesis.

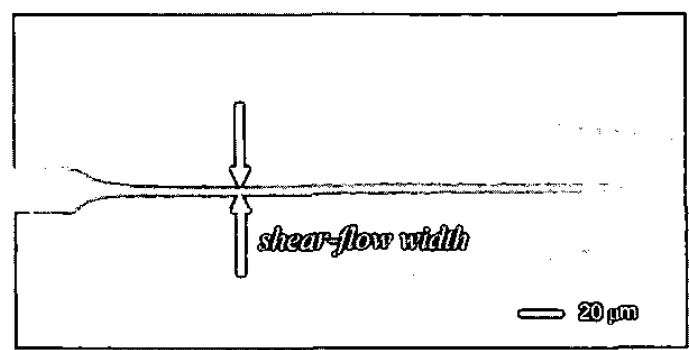

Figure 3: Shear-flow width calibration.

\section{Reaction system calibration}

The success of the proposed "muscle" fiber assembly depends on the conjugation reaction between the thiol groups of the "muscle" molecules and gold nanoparticles. To study the conjugation efficiency under various conditions, we have engineered special thiolated "model molecules" (bisdisulfide/ $\mathrm{C}_{28} \mathrm{H}_{34} \mathrm{O}_{4} \mathrm{~S}_{4}$ ) as shown in Figure 4. With model molecules we then study the solvent effects, gold nanoparticle size effects and temperature effects to find the working window for gold-molecule conjugation.

\section{Solvent effect}

First, we find that model molecules dissolve poorly in water but they dissolve much better in DCM $(>100 \mathrm{mM})$, acetone (about $1 \mathrm{mM})$ and ethanol $(<100 \mu \mathrm{M})$. On the other hand, the colloidal gold nanoparticle solution from British Bio-cell Inc. (BBI) is a water-based system. We therefore need to find a solvent that can dissolve reasonable amount of "muscle" molecules and won't destabilize gold nanoparticles when mixed with gold solution.

A screening experiment is then carried out to find a suitable solvent system. First we find that we could do solvent exchange for $30 \mathrm{~nm}$ gold nanoparticles. We centrifuge aqueous nano gold solution and re-suspend gold precipitate in ethanol, IPA and acetone. As negative controls, we mixed ethanol, IPA, acetone and DCM with gold solution based on water, ethanol, IPA and acetone. DCM is found to cause agglomeration of gold nanoparticles. IPA also destabilizes aqueous gold solution slowly. When model molecules in acetone are mixed with water, crash out of molecules happens as a result of low solubility. Finally, we choose model molecules in ethanol and gold nanoparticle in ethanol as the solvent system. As shown in Figure 5(a), upon mixing model molecule solution with gold solution the model molecules can crosslink gold nanoparticles to change solution color from red to purple/black and eventually the aggregate will precipitate [2]. This reaction is not shown in negative controls which do not contain molecules meaning that aggregation of gold is caused by molecule cross-linking of nanoparticles.

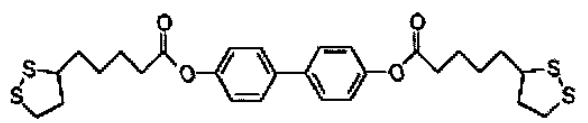

Figure 4: Bisdisulfide model molecule.

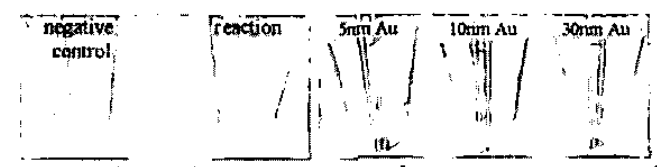

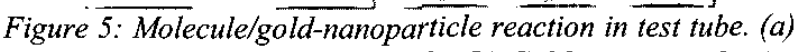
molecule/gold reaction in ethanol. (b) Gold nanoparticle size effect.

\section{Gold nanoparticle size effect}

Gold nanoparticle solutions with particle sizes of $5 \mathrm{~nm}$, $10 \mathrm{~nm}$ and $30 \mathrm{~nm}$ are used to study the particle size effect on gold-molecule conjugation. Figure $5(\mathrm{~b})$ shows that only $30 \mathrm{~nm}$ gold particle solution provides visible reaction. We believe this gold size effect is related to geometrical shapes of gold nanoparticles. Colloidal gold with a diameter larger than $25 \mathrm{~nm}$ exhibit geometrical eccentricity [3]. In fact, our TEM pictures (Figure 6) verify that $30 \mathrm{~nm}$ gold nanoparticles have crystalline nature. These crystal facets provide larger binding area/energy for molecules to bridge them and result in stable aggregate.

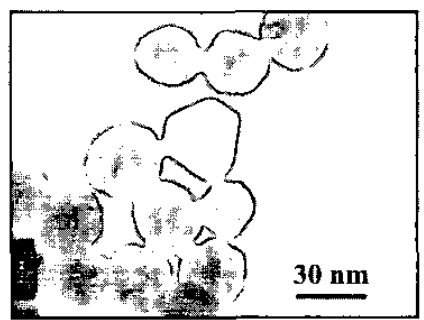

Figure 6: TEM of 30nm gold nanoparticles.

\section{Temperature effect}

Temperature treatment of the molecule-gold aggregate was performed to see if higher temperature can break the thiol-gold bonds. Reaction mixture of aggregates are heated up to $100^{\circ} \mathrm{C}$ for $2 \mathrm{hr}$. There is no visible change to the aggregate from our experiments meaning that thiol-gold bonds do not break in 
general up to $100^{\circ} \mathrm{C}$. This is positive in terms of the high stability of aggregates once formed.

\section{RESUltS AND ANALYSIS}

Self-assembly of aggregates made of gold nanoparticles and model molecules are carried out in a 4-channel input shear flow device as shown in Figure 7. Gold-molecule aggregates form right after shear flows meet. In the downstream, aggregates accumulate and continue to grow in size. TEM of the aggregates (Figure 8 ) clearly shows $3 \mathrm{D}$ cross-linking of the gold nanoparticles.

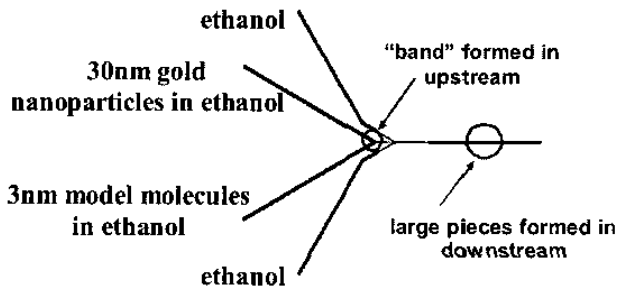

(a) Device configuration and experimental setup.

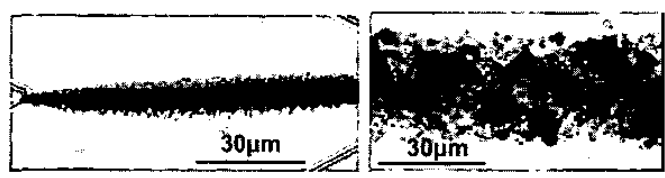

(b) Assembled "band" structure right after mixing of gold and molecule flow (left) and assembled structure confined by ethanol sheath flow in the downstream (right).

Figure 7: Molecule/gold-nanoparticles assembly in device.

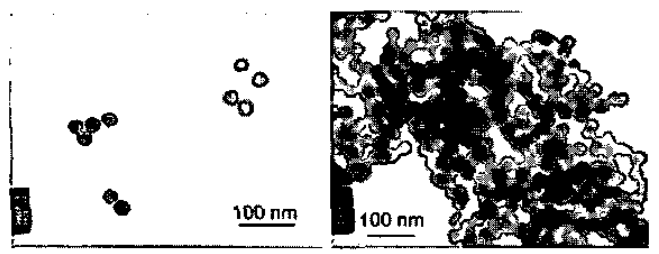

Figure8: TEM pictures. Pure $30 \mathrm{~nm}$ gold in water (left). Assembled structure in device (right).

\section{Aggregate size distribution}

With the formation of aggregates in the downstream of shear flow device, we further examine the size distribution of the aggregate particles. Figure 9(a) shows the distribution of the aggregate size over time. When the aggregation is done in test tubes we find that the aggregate particle sizes is quite homogeneous and in general smaller than $10 \mu \mathrm{m}$. On the other hand, when fluidic device is used to form the aggregates, significant improvement in terms of forming large size aggregates in a short time is achieved. First, the aggregate size continues to grow with time and up to $50 \mu \mathrm{m}$ size of aggregate has been found. Secondly, the growth rate in microfluidic device is found to be much faster than in the test tube reaction as shown in Figure 9(b). The results confirm the validity of our proposed shear-flow approach.

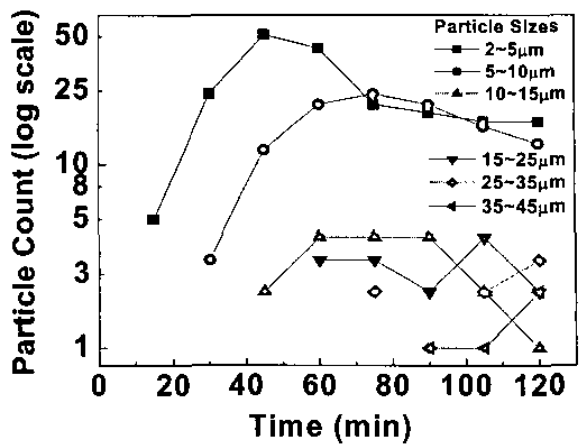

(a) Time evolution of particle size distribution in device.

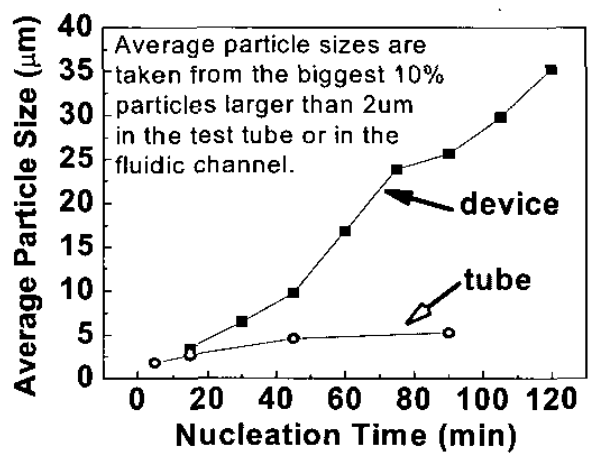

(b) Aggregate growth rate comparison.

Figure 9: Particle size and growth rate analysis.

\section{Electrical property of aggregates}

In order to study the electrical properties of as formed goldmolecule aggregates, pieces of aggregates are collected and carefully placed onto an electrode chip in liquid to bridge a pair of electrodes. A $20 \mathrm{hr}$ air dry followed by a $4 \mathrm{hr}$ baking at $100{ }^{\circ} \mathrm{C}$ is used to remove extra solvent and to form better electrical contact between the assembled material and the gold electrode as shown in Figure 10. I-V measurements show the aggregate formed by gold-molecule conjugation has a resistance of $16.1 \mathrm{G} \Omega$, which corresponds to a resistivity of $\sim$ $10^{6} \Omega$-cm based on the estimated size of the aggregate. In our experiments, we also do a control with only pure gold aggregate. Without surprise, pure gold aggregate has a very small resistivity of $\sim 10^{-5} \Omega-\mathrm{cm}$ because the aggregate is formed by gold-to-gold connection. The fact that goldmolecule aggregate has a resistivity 11 order-of-magnitude higher than the pure gold aggregate suggests that conjugation among gold nanoparticles and molecules is achieved in the assembly process. Large resistivity is due to the insulating molecules between gold nanoparticles. 
Furthermore, experiment also shows that no electrical breakdown was observed for gold-molecule aggregate up to $+/$ $100 \mathrm{~V}$ or $+/-40 \mathrm{kV} / \mathrm{cm}$, which is encouraging for the goal of using electrical signal to actuate motor molecule.
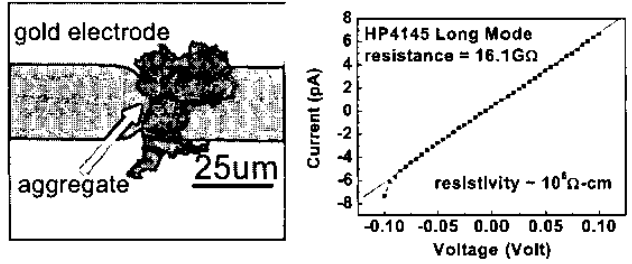

Figure 10: Electrical property measurement of molecule-gold aggregate.

\section{Aggregate collector}

In order to collect and shape the aggregate formed in the shear flow device, an in-channel collector is built. Figure 11 shows that a dense and rectangular-shaped aggregate is successfully collected. The leak holes around the collector are made to avoid aggregate clogging around the collector inlet.

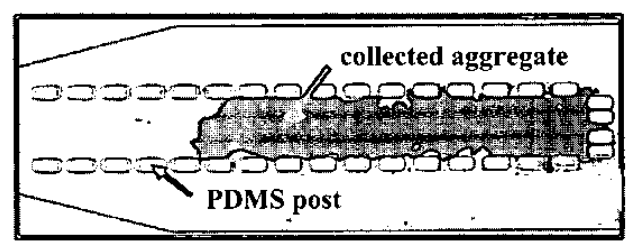

Figure 11: In-channel aggregate collector.

\section{Alternative assembly strategies}

The versatile use of shear-flow assembly is further demonstrated by two other examples. First, when longer or stiffer motor molecules are used to cross-link gold nanoparticles, conjugation reaction will need much longer time. In this case, "static" liquid interface can be created to

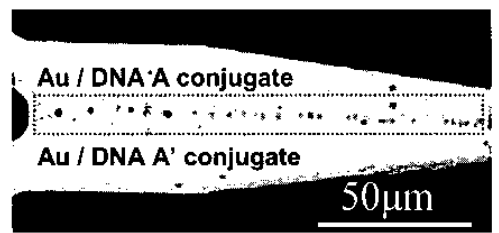

Figure 12: Gold nanoparticle assembly assisted by DNA hybridization.

serve the purpose. Figure 12 shows self-assembly of complementary gold/DNA conjugates through DNA hybridization [2] at the liquid interface 12 hours after selfassembly in the "stopped" shear flow channel. The second demonstration is to assemble high-aspect-ratio fibers. To do so, separate positively and negatively charged gold nanoparticle streams are flown into the two-input shear flow device as shown in Figure 13. Electrostatic attraction between the charged particles then quickly forms a $2 \mathrm{~mm}-\mathrm{long} / 12 \mu \mathrm{m}$ high fiber within seconds.

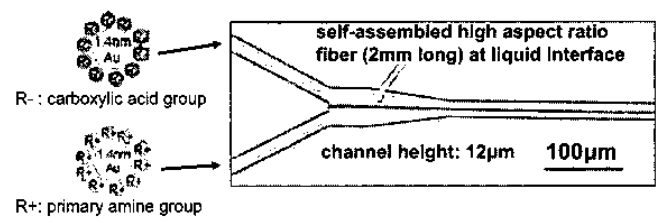

(a) Fiber/sheet structure formed along flow interface.

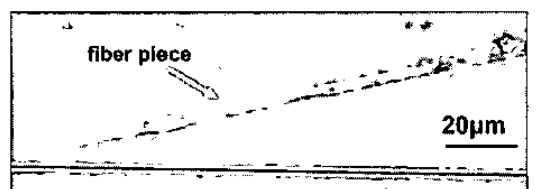

(b) Assembled fiber piece flushed to the downstream.

Figure 13: Sheet/Fiber structure formed by self-assembly of positively/negatively charged gold nanoparticles.

\section{Conclusions}

Molecular assembly using shear flow devices and thiolgold bonding chemistry is demonstrated. Gold-molecule aggregates as big as $50 \mu \mathrm{m}$ that are completely made of $30 \mathrm{~nm}$ gold nanoparticles and $3 \mathrm{~nm}$ model molecules can be produced using our approach. Currently, we are studying using rotaxane, the linear motor molecules, to form muscle fiber with the techniques developed in this work. Selective growth and anchoring of aggregate/muscle on electrodes for making actuators are also under investigation.

\section{ACKNOWLEDGEMENTS}

This work is sponsored by DARPA Biomolecular Motor (BMM) project and the NSF Center for Neuromorphic Engineering (CNSE) at Caltech.

\section{REFERENCES}

[1] V. Balzani, A. Credi, F.M. Raymo and J.F. Stoddart, Angew. Chem. Int. Ed. (2000), 39, 3348-3391.

[2] J.J. Storhoff, A.A. Lazarides, R.C. Mucic, C.A. Mirkin, R.L. Letsinger and G.C. Schatz, J. Am. Chem. Soc. (2000), 122, 4640-4650.

[3] M.A. Hayat, "Colloidal Gold: Principles, Methods, and Applications", Academic: San Diego, 1989; Vol 1. 